Min Zhang, Jin-Yuan Lin, Jing Liu and Ban-Feng Ruan*

\title{
The crystal structure of (E)-2-(4-((4-fluorobenzyl) oxy)styryl)-4,6-dimethoxybenzaldehyde, $\mathrm{C}_{24} \mathrm{H}_{21} \mathrm{FO}_{4}$
}

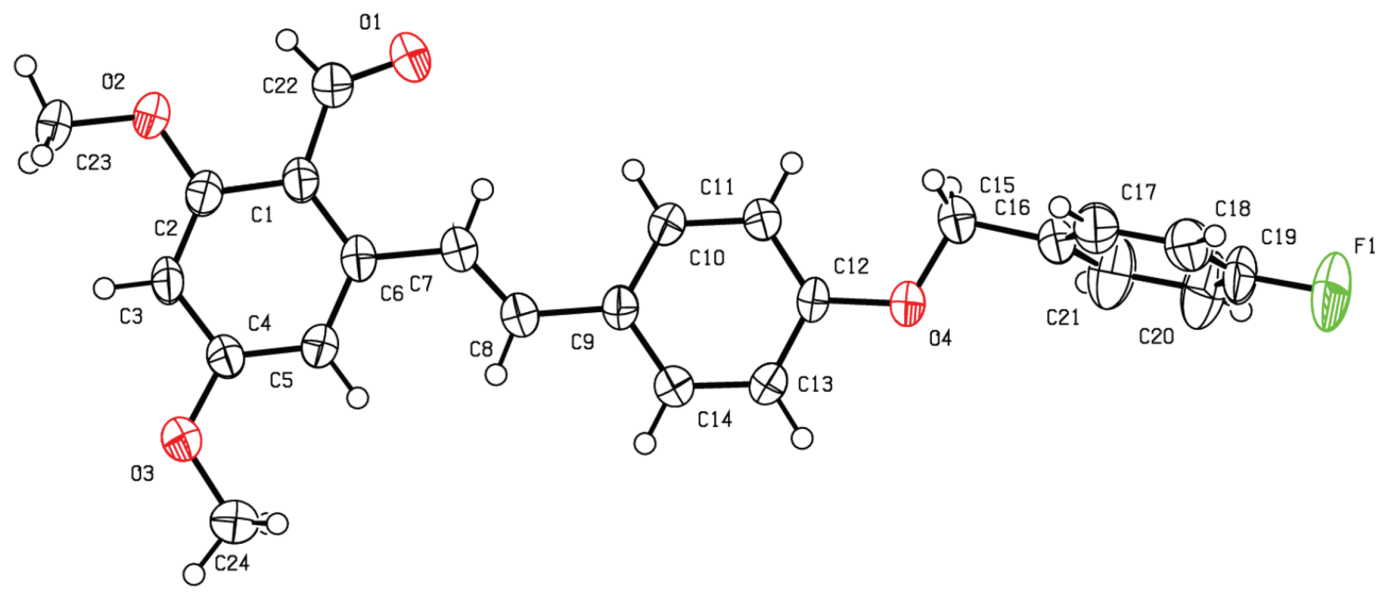

https://doi.org/10.1515/ncrs-2020-0168

Received April 1, 2020; accepted April 15, 2020; available online April 29, 2020

\begin{abstract}
$\mathrm{C}_{24} \mathrm{H}_{21} \mathrm{FO}_{4}, \quad$ triclinic, $\quad P \overline{1} \quad$ (no. 2), $a=6.9734(5) \AA$, $b=9.0402(7) \AA, \quad c=16.0963(12) \AA, \quad \alpha=98.977(6)^{\circ}$, $\beta=92.867(6)^{\circ}, \quad \gamma=97.501(6)^{\circ}, \quad V=991.14(12) \AA^{3}, \quad Z=2$, $R_{\mathrm{gt}}(F)=0.0540, w R_{\text {ref }}\left(F^{2}\right)=0.1406, T=273 \mathrm{~K}$.
\end{abstract}

CCDC no.: 1958969

The molecular structure is shown in the figure. Table 1 contains crystallographic data and Table 2 contains the list of the atoms including atomic coordinates and displacement parameters.

\section{Source of material}

To a stirred solution of (E)-1-(4-(4-fluorobenzyl)oxy)styryl)-3,5dimethoxybenzene (0.73 g, $2 \mathrm{mmol}$ ) [3], DMF (0.31 mL) in acetonitrile $(20 \mathrm{~mL})$ was added $\mathrm{POCl}_{3}(1.2 \mathrm{~mL})$ dropwise while cooling with an ice bath. The reaction mixture was stirred at

*Corresponding author: Ban-Feng Ruan, School of Biology, Food and Environment, Hefei University, Hefei 230601, P.R. China, e-mail: ruanbf@hfuu.edu.cn. https://orcid.org/0000-0002-98372237

Min Zhang, Jin-Yuan Lin and Jing Liu: School of Biology, Food and Environment, Hefei University, Hefei 230601, P.R. China

๑ Open Access. ( 2020 Min Zhang et al., published by De Gruyter. (c) BY International License.
Table 1: Data collection and handling.

\begin{tabular}{ll}
\hline Crystal: & Colourless block \\
Size: & $0.21 \times 0.17 \times 0.07 \mathrm{~mm}$ \\
Wavelength: & Mo $K \alpha$ radiation $(0.71073 \AA)$ \\
$\mu:$ & $0.10 \mathrm{~mm}^{-1}$ \\
Diffractometer, scan mode: & SuperNova, $\omega$ \\
$\theta_{\max }$, completeness: & $26.0^{\circ},>99 \%$ \\
$N(h k l)_{\text {measured }}, N(h k l)_{\text {unique }}, R_{\text {int }}:$ & $6688,3898,0.021$ \\
Criterion for $I_{\text {obs }}, N(h k l)_{\mathrm{gt}}:$ & $I_{\text {obs }}>2 \sigma\left(I_{\text {obs }}\right), 2598$ \\
$N(\text { param })_{\text {refined }}:$ & 264 \\
Programs: & CrysAlis \\
\hline
\end{tabular}

room temperature for $2 \mathrm{~h}$. After completion, the mixture was pured into ice water and neutralized by saturated aq. $\mathrm{NaOH}$. The mixture was stirred overnight and filtered to afford the crude product as yellow solid. After drying, the crude product was purified by column chromatography on silica gel to afford the title compound, using petroleum ether/ethylacetate (4:1, $v / v, \mathrm{R}_{f}=0.29$ ) as eluent. Crystals were obtained by slow evaporation from dichloromethane/methanol mixed solution.

\section{Experimental details}

All the non-hydrogen atoms were located in successive difference Fourier syntheses. The hydrogen atoms were added theoretically and riding on the concerned atoms.

\section{Comment}

Phenolic compounds make up one of the groups of molecules that have been most frequently studied in recent 
Table 2: Fractional atomic coordinates and isotropic or equivalent isotropic displacement parameters $\left(\AA^{2}\right)$.

\begin{tabular}{|c|c|c|c|c|}
\hline Atom & $x$ & $y$ & $z$ & $U_{\text {iso }} * / U_{\text {eq }}$ \\
\hline C1 & $0.5972(3)$ & $1.2267(2)$ & $0.10727(12)$ & $0.0379(4)$ \\
\hline C2 & $0.4099(3)$ & $1.1884(2)$ & $0.06510(12)$ & $0.0395(5)$ \\
\hline C3 & $0.3003(3)$ & $1.0513(2)$ & $0.06741(12)$ & $0.0414(5)$ \\
\hline H3 & 0.1775 & 1.0275 & 0.0395 & $0.050^{\star}$ \\
\hline C4 & $0.3739(3)$ & $0.9491(2)$ & $0.11153(12)$ & $0.0398(5)$ \\
\hline C5 & $0.5543(3)$ & $0.9838(2)$ & $0.15437(12)$ & $0.0404(5)$ \\
\hline H5 & 0.5999 & 0.9147 & 0.1849 & $0.048^{\star}$ \\
\hline C6 & $0.6693(3)$ & $1.1215(2)$ & $0.15246(12)$ & $0.0379(4)$ \\
\hline C7 & $0.8611(3)$ & $1.1553(2)$ & $0.19862(13)$ & $0.0448(5)$ \\
\hline H7 & 0.9244 & 1.2533 & 0.2017 & $0.054^{*}$ \\
\hline C8 & $0.9530(3)$ & $1.0644(2)$ & $0.23565(13)$ & $0.0492(5)$ \\
\hline H8 & 0.8916 & 0.9653 & 0.2302 & 0.059 * \\
\hline C9 & $1.1402(3)$ & $1.0978(2)$ & $0.28477(12)$ & $0.0402(5)$ \\
\hline C10 & $1.2654(3)$ & $1.2314(2)$ & $0.28871(12)$ & $0.0415(5)$ \\
\hline $\mathrm{H} 10$ & 1.2343 & 1.3020 & 0.2558 & $0.050^{\star}$ \\
\hline C11 & $1.4351(3)$ & $1.2625(2)$ & $0.34013(12)$ & $0.0440(5)$ \\
\hline H11 & 1.5165 & 1.3531 & 0.3418 & $0.053^{*}$ \\
\hline C12 & $1.4836(3)$ & $1.1584(2)$ & $0.38909(12)$ & $0.0398(5)$ \\
\hline C13 & $1.3651(3)$ & $1.0220(2)$ & $0.38363(14)$ & $0.0501(6)$ \\
\hline H13 & 1.3984 & 0.9498 & 0.4150 & $0.060^{\star}$ \\
\hline C14 & 1.1985(3) & $0.9938(2)$ & $0.33183(14)$ & $0.0516(6)$ \\
\hline H14 & 1.1212 & 0.9008 & 0.3281 & $0.062^{\star}$ \\
\hline C15 & $1.7612(3)$ & $1.3210(2)$ & $0.45948(15)$ & $0.0613(7)$ \\
\hline H15A & 1.6841 & 1.4018 & 0.4746 & $0.074^{*}$ \\
\hline H15B & 1.8226 & 1.3369 & 0.4082 & $0.074^{\star}$ \\
\hline C16 & $1.9124(3)$ & $1.3218(2)$ & $0.52927(14)$ & $0.0484(5)$ \\
\hline C17 & $1.8995(3)$ & $1.3974(2)$ & $0.60911(15)$ & $0.0574(6)$ \\
\hline H17 & 1.7925 & 1.4466 & 0.6207 & $0.069^{\star}$ \\
\hline C18 & $2.0418(4)$ & $1.4022(3)$ & $0.67276(15)$ & $0.0633(7)$ \\
\hline H18 & 2.0318 & 1.4539 & 0.7267 & $0.076^{\star}$ \\
\hline C19 & $2.1950(3)$ & 1.3303(3) & $0.65446(17)$ & $0.0675(7)$ \\
\hline C20 & $2.2142(4)$ & $1.2535(4)$ & $0.57634(19)$ & $0.0880(9)$ \\
\hline $\mathrm{H} 2 \mathrm{O}$ & 2.3218 & 1.2046 & 0.5654 & $0.106^{\star}$ \\
\hline C21 & $2.0708(3)$ & $1.2497(3)$ & $0.51377(16)$ & $0.0743(8)$ \\
\hline $\mathrm{H} 21$ & 2.0818 & 1.1973 & 0.4601 & $0.089^{\star}$ \\
\hline C22 & $0.7051(3)$ & $1.3726(2)$ & $0.09843(13)$ & $0.0462(5)$ \\
\hline $\mathrm{H} 22$ & 0.6400 & 1.4313 & 0.0671 & $0.055^{\star}$ \\
\hline $\mathrm{C} 23$ & $0.1566(3)$ & $1.2617(3)$ & $-0.01881(15)$ & $0.0554(6)$ \\
\hline $\mathrm{H} 23 \mathrm{~A}$ & 0.1489 & 1.1728 & -0.0608 & $0.083^{*}$ \\
\hline H23B & 0.1299 & 1.3457 & -0.0452 & $0.083^{*}$ \\
\hline $\mathrm{H} 23 \mathrm{C}$ & 0.0632 & 1.2447 & 0.0218 & $0.083^{*}$ \\
\hline C24 & $0.3203(3)$ & $0.7009(2)$ & $0.14818(16)$ & $0.0627(7)$ \\
\hline $\mathrm{H} 24 \mathrm{~A}$ & 0.4383 & 0.6757 & 0.1250 & $0.094^{\star}$ \\
\hline H24B & 0.2236 & 0.6129 & 0.1384 & $0.094^{\star}$ \\
\hline $\mathrm{H} 24 \mathrm{C}$ & 0.3437 & 0.7358 & 0.2077 & $0.094^{\star}$ \\
\hline F1 & $2.3377(2)$ & $1.3340(2)$ & $0.71552(11)$ & $0.1143(7)$ \\
\hline 01 & $0.8673(2)$ & $1.42731(16)$ & $0.12631(10)$ & $0.0588(4)$ \\
\hline 02 & $0.34692(19)$ & $1.29427(15)$ & $0.02269(10)$ & $0.0551(4)$ \\
\hline 03 & $0.25379(19)$ & $0.81695(15)$ & $0.10873(10)$ & $0.0550(4)$ \\
\hline 04 & $1.64147(19)$ & $1.17957(15)$ & $0.44637(9)$ & $0.0530(4)$ \\
\hline
\end{tabular}

years [4]. Among them, pterostilbene (trans-3,5-dimethoxy$4^{\prime}$-hydroxystilbene) is a natural dimethyl ether analogue to resveratrol, which is classified as a phytoalexin [3]. Up to now, pterostilbene is found to have diverse biological benefits in the prevention and treatment of many diseases, such as anticancer [5-7], anti-inflammation [8-10], anti-diabetes [11], the prevention of cardiovascular and congitive function degeneration [12]. Consequently, pterostilbene has good potential to be evaluated for therapeutic use.

However, pterostilbene as a natural compound in commercial products has been problematic due to its rapid metabolism, especially when taken orally [13]. Thus, many researchers have attempted to optimize or modify the structures of pterostilbenes in order to achieve better pharmacokinetic characteristics and bioavailability. In our group, we have been working on this subject for many years and diversified derivatives containing pterostilbene have been degigned and synthesized [14, 15]. Currently, we have found that pterostilbene-based nitrovinylstilbenes exhibited potential NLRP3 inflammasome activation inhibitory activity. This indicates that this kind of compounds could be candidates to discovery new drug for treatment of inflammation-related diseases. During this process, we have optimized the synthetic route of the key intermediate reported here and characterized its structure by single crystal diffraction.

The title compound crystallizes in the triclinic space group $P \overline{1}$. There is one title molecule in the asymmetric unit (see the figure). The C7-C8 bond length [1.3013(30) $\AA$ ] is consistent with the expected value for a $\mathrm{C}=\mathrm{C}$ double bond. Similarly, the C22-01 bond length [1.208(23) $\AA$ ] is consistent with the expected value for a carbonyl $\mathrm{C}-\mathrm{O}$ double bond. The angles of $\mathrm{C} 6-\mathrm{C} 7-\mathrm{C} 8$ and $\mathrm{C} 7-\mathrm{C} 8-\mathrm{C} 9$ are $127.7(2)^{\circ}$ and $128.4(2)^{\circ}$ respectively, indicating that it has the $E$ configuration (see the figure). All bond lengths and angles are within normal ranges to previously reported structures [16].

Acknowledgements: This work was supported by the Anhui quality engineering project: Experimental training center project/Award (No: 2016sxzx036) and the Anhui Province University Natural Science Research Project (No: KJ2019A0824).

\section{References}

1. Oxford Diffraction: CrysAlisPRO, Oxford Diffraction Ltd, Abingdon, Oxfordshire, England (2006).

2. Sheldrick, G. M.: SHELXT - Integrated space-group and crystalstructure determination. Acta Crystallogr. A71 (2015) 3-8.

3. Gomez-Zorita, S.; Fernandez-Quintela, A.; Lasa, A.; Aguirre, L.; Rimando, A. M.; Portillo, M. P.: Pterostilbene, a dimethyl ether derivative of resveratrol, reduces fat accumulation in rats fed an obesogenic diet. J. Agric. Food Chem. 62 (2014) 8371-8378.

4. Chen, L.-Z.; Li, Y.; Jiao, M.-M.; Shi, J.-B.; Tan, Y.; Ruan, B.-F.; Liu, X.-H.: Novel resveratrol-based flavonol derivatives: synthesis and anti-inflammatory activity in vitro and in vivo. Eur. J. Med. Chem. 175 (2019) 114-128. 
5. Rimando, A. M.; Suh, N.: Biological/chemopreventive activity of stilbenes and their effect on colon cancer. Planta Med. 74 (2008) 1635-1643.

6. Jung, J.-H.; Shin, E.-A.; Kim, J.-H.; Sim, D.-Y.; Lee, H.; Park, J.-E.; Lee, H.-J.; Kim, S.-H.: NEDD9 inhibition by miR-25-5p activation is critically involved in co-treatment of melatonin- and pterostilbene-induced apoptosis in colorectal cancer cells. Cancers 11 (2019) 1684.

7. Hsiao, Y.-H.; Chen, N.-C.; Koh, Y.-C.; Nagabhushanam, K.; Ho, C.-T.; Pan, M.-H.: Pterostilbene inhibits adipocyte conditionedmedium-induced colorectal cancer cell migration through targeting FABP5-related signaling pathway. J. Agric. Food Chem. 67 (2019) 10321-10329.

8. Kosuru, R.; Kandula, V.; Rai, U.; Prakash, S.; Xia, Z.-Y.; Singh, S.: Pterostilbene decreases cardiac oxidative stress and inflammation via activation of AMPK/Nrf2/HO-1 pathway in fructose-fed diabetic rats. Cardiovasc. Drugs Ther. 32 (2018) 147-163.

9. Xue, E. X.; Lin, J.-P.; Zhang, Y.; Sheng, S.-R.; Liu, H.-X.; Zhou, Y.L.; Xu, H.: Pterostilbene inhibits inflammation and ROS production in chondrocytes by activating Nrf2 pathway. Oncotarget 8 (2017) 41988-42000.

10. Zhang, Y.; Zhang, Y.: Pterostilbene, a novel natural plant conduct, inhibits high fat-induced atherosclerosis inflammation via NF-kB signaling pathway in Toll-like receptor
5 (TLR5) deficient mice. Biomed. Pharmacother. 81 (2016) 345-355.

11. Satheesh, M. A.; Pari, L.: The antioxidant role of pterostilbene in streptozotocin-nicotinamide induced type 2 diabetes mellitus in Wistar rats. J. Pharm. Pharmacol. 58 (2006) 1483-14904.

12. Joseph, J. A.; Fisher, D. R.; Cheng, V.; Rimando, A. M.; ShukittHale, B.: Cellular and behavioral effects of stilbene resveratrol analogues: implications for reducing the deleterious effects of aging. J. Agric. Food Chem. 56 (2008) 10544-10551.

13. Wang, P.; Sang, S.-M.: Metabolism and pharmacokinetics of resveratrol and pterostilbene. BioFactors 44 (2018) 16-25.

14. Ruan, B.-F.; Ge, W.-W.; Cheng, H.-J.; Xu, H.-J.; Li, Q.-S.; Liu, X.-H.: Resveratrol-based cinnamic ester hybrids: synthesis, characterization, and anti-inflammatory activity. J. Enzyme Inhib. Med. Chem. 32 (2017) 1282-1290.

15. Ruan, B.-F.; Cheng, H.-J.; Ren, J.; Li, H.-L.; Guo, L.-L.; Zhang, X.-X.; Liao, C.-Z.: Novel $2 \mathrm{H}$-chromen-2-one derivatives of resveratrol: design, synthesis, modeling and use as human monoamine oxidase inhibitors. Eur. J. Med. Chem. 103 (2015) 185-190.

16. Yao, R.-S.; Lu, X.-Q.; Guan, Q.-X.; Zheng, L.; Lu, X.; Ruan, B.-F.: Novel $2 \mathrm{H}$-chromen-2-one derivatives of resveratrol: design, synthesis, modeling and use as human monoamine oxidase inhibitors. Eur. J. Med. Chem. 62 (2013) 222-231. 